\title{
Field-of-View Prediction in 360-Degree Videos with Attention-based Neural Encoder-Decoder Networks
}

\author{
Jiang Yu \\ New York University \\ jiangyu@nyu.edu
}

\begin{abstract}
In this paper, we propose attention-based neural encoder-decoder networks for predicting user Field-of-View (FoV) in 360-degree videos. Our proposed prediction methods are based on the attention mechanism that learns the weighted prediction power of historical FoV time series through end-to-end training. Attention-based neural encoder-decoder networks do not involve recursion, thus can be highly parallelized during training. Using publicly available 360-degree head movement datasets, we demonstrate that our FoV prediction models outperform the state-of-art FoV prediction models, achieving lower prediction error, higher training throughput, and faster convergence. Better FoV prediction leads to reduced bandwidth consumption, better video quality, and improved user quality of experience.
\end{abstract}

\section{CCS CONCEPTS}

- Information systems $\rightarrow$ Multimedia streaming; • Computing methodologies $\rightarrow$ Machine learning approaches; Neural networks.

\section{KEYWORDS}

field of view prediction, 360 degree videos, neural networks, encoder decoder networks, attention

\section{ACM Reference Format:}

Jiang Yu and Yong Liu. 2019. Field-of-View Prediction in 360-Degree Videos with Attention-based Neural Encoder-Decoder Networks. In 11th International Workshop on Immersive Mixed and Virtual Environment Systems (MMVE '19), June 21, 2019, Amherst, MA, USA. ACM, New York, NY, USA, 6 pages. https://doi.org/10.1145/3304113.3326118

\section{INTRODUCTION}

Immersive technologies, such as Virtual Reality (VR) and Augment Reality (AR), are becoming more and more important parts across different fields, including entertainment, education, health-care and etc. Among them, 360-degree video is one of the most attractive applications. It provides users with an immersive experience while watching videos. However, in order to offer a smooth and highquality watching experience, 360-degree video raises a big challenge for the network capability. Results in [11] show that to support

Permission to make digital or hard copies of all or part of this work for personal or classroom use is granted without fee provided that copies are not made or distributed for profit or commercial advantage and that copies bear this notice and the full citation on the first page. Copyrights for components of this work owned by others than ACM must be honored. Abstracting with credit is permitted. To copy otherwise, or republish, to post on servers or to redistribute to lists, requires prior specific permission and/or a fee. Request permissions from permissions@acm.org.

MMVE '19, fune 21, 2019, Amherst, MA, USA

(C) 2019 Association for Computing Machinery.

ACM ISBN 978-1-4503-6299-3/19/06 . \$ \$15.00

https://doi.org/10.1145/3304113.3326118

\author{
Yong Liu \\ New York University \\ yongliu@nyu.edu
}

fluent display of the "Entry-Level" 360-degree videos which have higher resolutions, network bandwidth should be at least $100 \mathrm{Mbps}$ and the round trip time (RTT) cannot be larger than $30 \mathrm{~ms}$. The current network capability is far from enough to provide users with a high-quality immersive video experience.

Even though 360-degree videos allow users to watch along any direction, at any specific time, only a certain FoV within a 360degree video is watched by a user. In other words, except for the content within the user's current FoV, all the other parts of the video have no effect on the final user Quality-of-Experience (QoE). Based on this observation, several methods have been proposed to address the problem of network bandwidth shortage. One common method is to transmit and pre-fetch only the video inside user FoV with the help of user FoV prediction. In this case, how well the user FoV prediction algorithm performs will affect the final 360-degree video quality significantly. Boldly, assuming there is a prediction algorithm which could predict user's future FoV sequence perfectly, the 360 video streaming problem becomes much easier and the video inside user FoV could be streamed in the same way as the traditional 2D planar video.

FoV prediction can be treated as a multivariate time series prediction problem. It predicts a future sequence of FoVs given a user's historical sequence of FoV trace. A variety of approaches have been proposed to increase the FoV prediction accuracy, including linear or polynomial regressions, recurrent neural networks, video content analysis, etc. In this work, we use attention-based encoder-decoder networks for the FoV prediction in 360-degree video, which enjoy both superior prediction accuracy and fast training performance. The main contributions of this paper are the following:

- We propose using novel Attention-based Neural EncoderDecoder Networks for FoV prediction. They better capture the inherent temporal patterns of user FoV changes than simple regression models and recurrent neural networks, resulting better prediction accuracy.

- Attention-based Neural Encoder-Decoder networks do not involve expensive recursion, their training can be parallelized and thus is much faster than recurrent neural networks.

- Using publicly available 360-degree head movement datasets, we demonstrate that the proposed model outperforms the state-of-art FoV prediction models, achieving lower prediction error, higher training throughput, and faster convergence.

\section{RELATED WORK}

FoV prediction falls into the category of sequence prediction, which is to predict a sequence of future values given a sequence of past values. Recently neural network based approaches have been proven to 
excel in a variety of sequence prediction problems [1][16]. We first briefly survey neural network based sequence prediction literature and then review a list of recent FoV prediction models.

Recurrent neural networks (RNNs) can be used for processing sequences, either as input, output or both. However, RNNs are wellknown to be hard to train when there are long-term dependencies in the data due to the vanishing gradient problem [5]. Some variations of it, such as Long Short-Term Memory (LSTM) networks [10] and Gated Recurrent Units (GRU)[6], have been developed to solve the problems and thus are effective for sequence prediction tasks. Based on LSTM, [12] uses a multi-layer LSTM to map an input sequence to a vector of a fixed dimension, and then uses another deep LSTM to decode the target sequence from the vector, which is widely known as the encoder-decoder architecture. [3] introduces an attention mechanism to further improve the performance of the encoder-decoder approach for problems with long-term dependencies. LSTMs and attention mechanism have been shown to achieve impressive performance in sequence prediction problems, such as human trajectory prediction [1], and predictive view generation for mobile 360-degree and VR experiences [16].

Most recently, researchers started to work on FoV prediction for 360-degree videos. Based on linear regression, [17] exploits cross-users behaviors for FoV prediction in 360-degree video and concurrently considers user's personalized information and information about cross-users behaviors to predict future FoV. In [4], a LSTM neural network is used to achieve higher accuracy over linear regression in FoV prediction. Most recently, [16] uses LSTM with tiling for predictive view generation and achieves the new stateof-the art FoV prediction performance. [15] combines features extracted from image contents and saliency maps with Convolutional Neural Network (CNN) and features encoded from view path history for gaze displacement prediction. However, linear regression based methods suffer from poor prediction performance, and LSTM based models suffer from slow training and inference performance. In this paper, we address these two issues with attention-based neural encoder-decoder networks. Attention-based encoder-decoder networks can better capture the inherent temporal patterns of FoV changes than simple regression models and recurrent neural networks, and they do not involve expensive recursion, thus their training can be parallelized and thus much faster than recurrent neural networks. The details are in the following sections.

\section{ATTENTION BASED NEURAL ENCODER-DECODER NETWORKS}

A 360 degree video display headset usually has three degrees of freedom for rotational head movements ( $y a w$, pitch and roll), or six degrees of freedom (transitional movements in $x, y, z$ in addition to the three angles). Due to delivery platform and HMD technologies restrictions [7, 8], two datasets out of the three datasets used in the paper only captured rotational head movements. Thus in this paper, we will only focus on the rotational head movements and ignore the transitional movements. Usually, the reference position (i.e. the $(\mathrm{O}, \mathrm{i}, \mathrm{j}, \mathrm{k})$ basis) is set at boot time by the head mounted device (HMD). $k$ is always vertical but $i$ and $j$ can change each time the HMD restarts. Between two reboots the reference position never changes [7]. According to the Euler's rotation theorem, any rotation

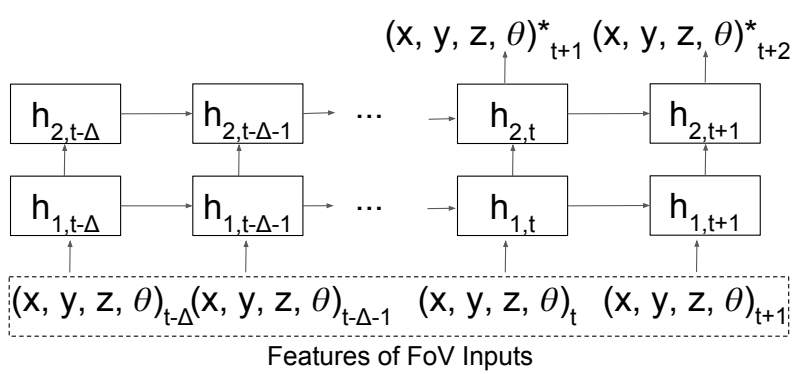

Figure 1: LSTM Model for FoV Prediction

or sequence of rotations of a three-dimensional coordinate system with fixed origin is equivalent to a single rotation around an axis, represented by a unit vector $u=(x, y, z)=x i+y j+z k$ in $R^{3}$, and by a given angle $\theta$, using the right hand rule. The rotational position of FoV can therefore be modeled by the four-tuple of $v_{t}=(x, y, z, \theta)_{t}$, where $t$ is the time step index.

The FoV prediction problem can be defined as a multivariate time series prediction problem. Given a sequence of past FoV values $v_{1}$, $v_{2}, \ldots, v_{T}$ of length $T$, we want to predict a sequence of future FoV values $v_{T+1}, v_{T+2}, \ldots, v_{T+F}$ of length $F$. In the following, we first introduce a strong baseline LSTM model and an improved LSTM model with attention, and then develop attention based-neural encoder-decoder networks for predicting FoV in 360-degree videos.

\subsection{Baseline: LSTM Model}

LSTM networks do well in learning the temporal dependence between different time steps due to their gated memory design [10]. We exploit this nice property of LSTM and design a multi-variate multi-layer LSTM network to serve as a strong baseline to learn users' viewpoint motion patterns and predict future FoV based on their past movements.

Figure 1 shows a two-layer LSTM network used in predicting FoV. The dimensions of the input values are dependent on the specific FoV datasets. The input dimension is four for datasets with the axis-angle FoV representation. The number of hidden layers is two and the hidden dimension of a LSTM unit is 128. The output dimensions are the same as input dimensions.

The model takes a normalized vector of FoV value tuples as feature inputs one at a time. After having finished processing all inputs, it then predicts a vector of four normalized values of FoV for the first time step into the future given the hidden state after inputting the previous sequence of FoV features. Then, it feeds back the predicted values to the LSTM model to update its hidden state and continues to predict the next step future values. The process is repeated until all $F$ predictions are completed. The model learns its parameters by minimizing the mean squared error between a sequence of predicted FoV values in the output and the target ground-truth values.

\subsection{LSTM Model with Attention}

LSTM models are proven to be effective in sequence prediction tasks. However, the prediction performance can be further improved by 


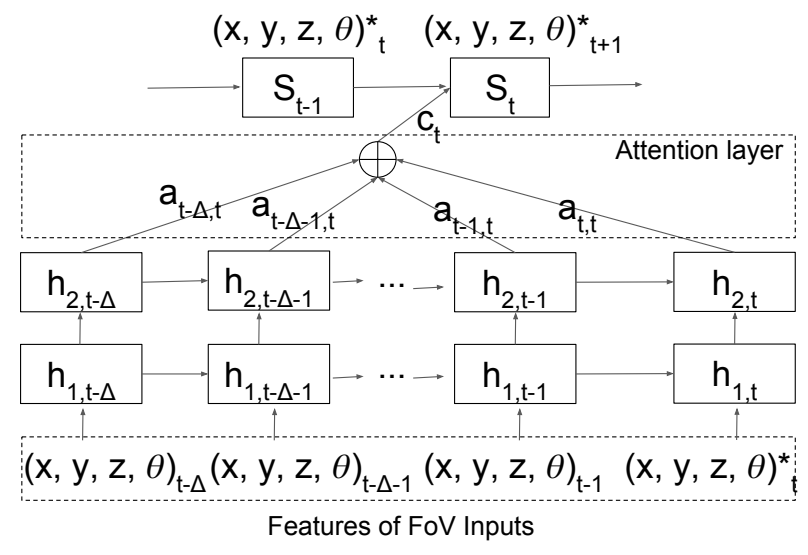

Figure 2: LSTM with Attention

incorporating the attention mechanism. Attention has been established as an important part of compelling sequence modeling in various tasks [13]. Most competitive neural sequence models have an encode-decoder structure. We follow this general rule in designing a LSTM model with Attention for FoV prediction. The encoder maps an input sequence of FoV traces $\left(v_{1}, v_{2}, \ldots, v_{n}\right)$ into a sequence of continuous abstract representation $\left(h_{1}, h_{2}, \ldots, h_{n}\right)$. The decoder then generates a sequence of future FoV outputs one step at a time. The decoding process is auto-regressive and uses the prediction outputs as additional inputs when predicting the next one.

With attention, we no longer need to encode the full input sequence into a fixed-length vector. This is unlike the traditional encoder-decoder which limits the capacity of the expressiveness of the model. Rather, the attention allows the decoder to attend to different most relevant parts of the input sequence at each step of the output generation. More importantly, the model is trained to learn what to attend to based on the input sequence and what it has output so far.

The LSTM with attention network architecture and settings are similar to the LSTM models in Figure 1 except for the added attention layer as shown in Figure 2. The attention layer consists of $\Delta$ attention weights $\alpha_{i, t}$ at each time step, and its hidden dimension has size of 256, where $t-\Delta \leq i<t$. They are used to compute a context vector $C_{t}$ at each time step $t$. The usage is as follows.

The output conditional probability is approximated by a neural network $g$ as follows,

$$
p\left(v_{T+i}^{*} \mid v_{1}, v_{2}, \ldots, v_{T}, v_{T+i-1}^{*}\right)=g\left(v_{T+i-1}^{*}, s_{T+i-1}, c_{T+i-1}\right),
$$

where $i=1, \ldots, F$, and $S_{i}$ is the output state for time $i$ and is computed by,

$$
S_{i}=f\left(S_{i-1}, C_{i}\right)
$$

The context vector $C_{t}$ is computed as a weighted sum of the hidden states,

$$
C_{i}=\sum_{j=t-\Delta}^{t} \alpha_{i, j} h_{j}
$$

The attention weight $\alpha_{i, j}$ is computed as,

$$
\alpha_{i, j}=\frac{\exp \left(e_{i, j}\right)}{\sum_{k=1}^{T} \exp \left(e_{i, k}\right)}
$$

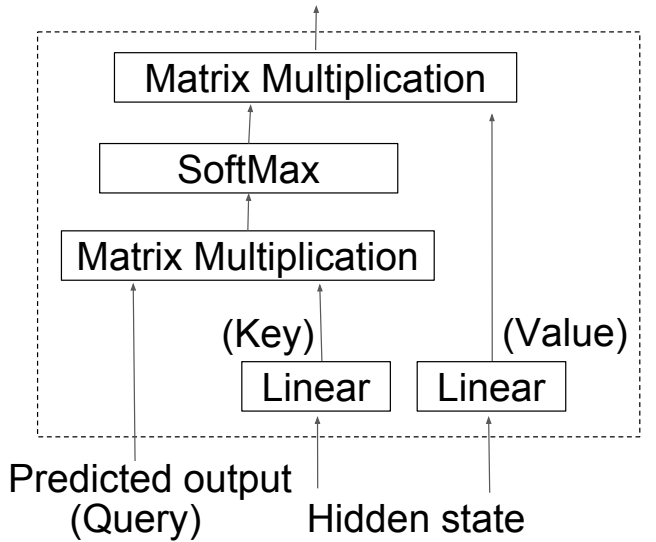

Figure 3: Attention Mechanism

where $e_{i, j}$ is an alignment model which scores how well the inputs around the position $j$ and the outputs around the position $i$ match. It is computed as,

$$
e_{i, j}=f_{a}\left(S_{i-1}, h_{j}\right)
$$

where $t \leq i \leq t+F, t-\Delta \leq j<t$. The alignment model $f_{a}(\cdot)$ is parameterized as a feed-forward neural network which is jointly trained with all the other components of the prediction model.

\subsection{Attention-based Encoder-Decoder Networks}

LSTM with attention can improve the prediction performance. However, LSTMs are notoriously slow to train due to their inherently sequential training process. What's more, adding attention on top of LSTM further increases its computational complexity, the training process become even slower. Especially in online learning settings, FoV user trace data is generated and transmitted in a streaming fashion, training throughput and speed become critical. Faster training enables feeding more recent data into the training process, and leaves more time for video transmission.

To address the slow training problem and further improve accuracy, we introduce an attention-based encoder-decoder model whose training can be parallelized as in [13]. They can better capture the inherent temporal patterns of FoV changes than simple regression models and recurrent neural networks such as LSTM, leading to better prediction accuracy. More importantly, the networks do not have expensive recursion, the training can be fully parallelized and thus will be much faster than recurrent neural networks. The attention-based architecture consists of two major parts: Encoder network and Decoder network, and its details are broken down as follows.

3.3.1 Attention Mechanism. Firstly, we describe attention mechanism used in the model. As illustrated in Figure 3, our attention model takes as input a query and a set of key-value pairs, returns a weighted sum of the values as an output. The weights are called attention weight which indicates the compatibility between the query and keys. In the Encoder network, the hidden state serves as the inputs for query, key and values pair. In the Decoder network, the hidden state serves as the inputs for key and value pair 


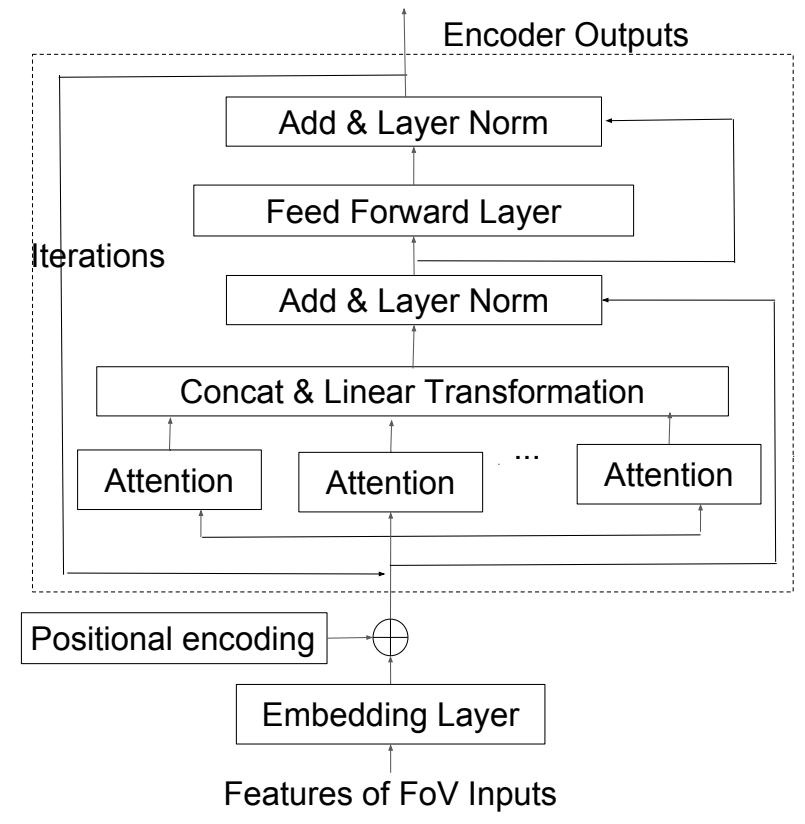

Figure 4: Attention-based Encoder

while the previous predicted outputs is query. We use four parallel attention layers to learn a better representation of the inputs. The attention is a mechanism to allow the model to better predict next value based on the context of input values and the condition of outputted values.

3.3.2 Encoder Network. The Encoder Network consists of two hidden layers. Each layer has an attention mechanism, followed by a layer of normalization, and then a fully connected feed forward network followed by a second layer of normalization. Two hidden layers are stacked together, with residual connections around each layer and attention and FFN. It has 2 hidden layers, with hidden size of 128 and uses filters of size 512. The number of attention heads is 4. The encoder of the attention predictor is illustrated in Figure 4.

The learned embeddings are used to convert the FoV inputs and outputs to vectors of dimension $h$, which is a common practice in sequence to sequence models. Besides that, learned linear transformation and the softmax function are used to convert the decoder output to predict the probabilities of the next value. The weight matrix between the two embedding layers and the pre-softmax linear transformation are shared.

Due to the lack of recurrence and convolution of attention based model, we need to inject some information about the relative position of the inputs in the sequence for the model to utilize the order information of the sequence. We add sine and cosine functions of different frequencies as proposed by [13] to make the model aware of the position of each input value. Fully connected feed forward networks are used after the attention layer. There are two linear transformations and a nonlinear activation in between. We use residual connection [9] between each layer to increase the flexibility and capacity of the model. We also use layer normalization [2] to stabilize the training and thus increase the convergence.

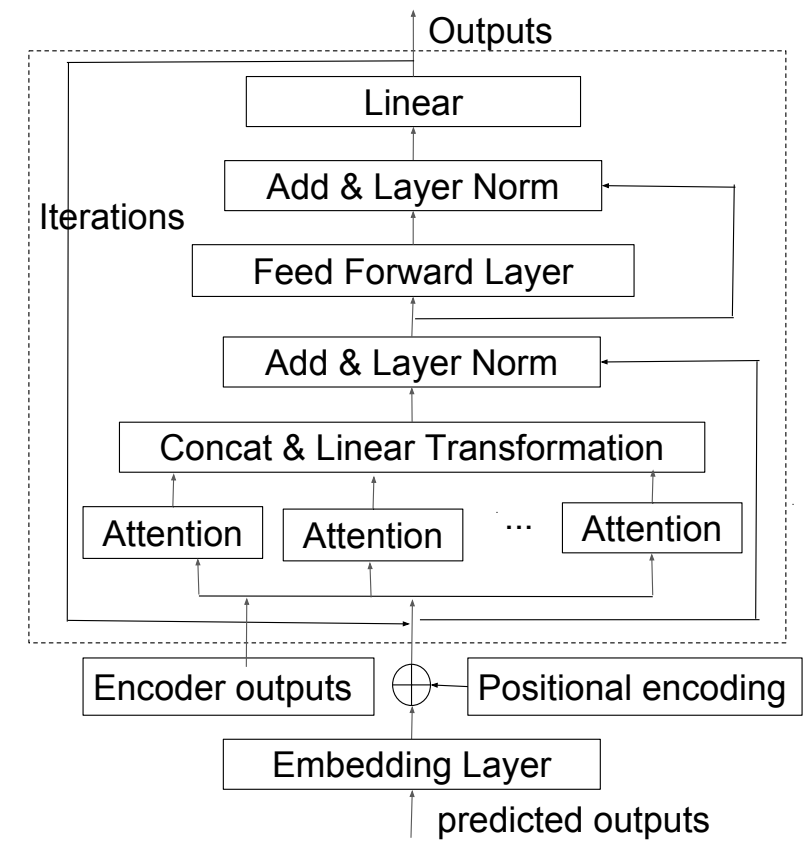

Figure 5: Attention-based Decoder

3.3.3 Decoder Network. Similarly, the decoder shown in Figure 5 consists of two stacked identical layers. In addition to the two sub-layers of attention and FFN, it also has a third attention layer which attends to the output of the encoder network. The sizes of hidden layers, filters, and number of attention heads of decoder part are the as the Encoder Network.

One important distinction from the encoder network is the inference steps. When predicting $F$ future FoV values, the decoder network firstly predicts the first future value, and then feeds back the previous prediction into the network as query of the attention mechanism to do a conditional generation of the next future FoV values. It repeats until all $F$ future FoV values are generated.

3.3.4 Optimizer. We use Adam as optimizer. For the attentionbased model, we use batch size of 256, and learning rate is set as the following decay function with warm up steps of 1,800 and learning rate constant of 1.8 .

$$
\text { initConstant } * \text { linearWarmup } * \frac{1}{\sqrt{\text { decay }}} * \frac{1}{\sqrt{\text { hiddenSize }}}
$$

\section{EXPERIMENTS}

\subsection{Datasets and Setup}

We evaluate and compare the Attention-based Encoder-Decoder model, LSTM and LSTM with attention models in this section, by running experiments on the three publicly available 360 degree video head movement datasets $[7,8,14]$.

The NYU dataset was collected by recording the positions of the video displayed on computer screen in 360 degree videos when viewers watch them. The viewers could drag the screen up or down, left or right when watching the 360 degree videos. It contains user screen movement traces of about 47 users watching 12 short 360 


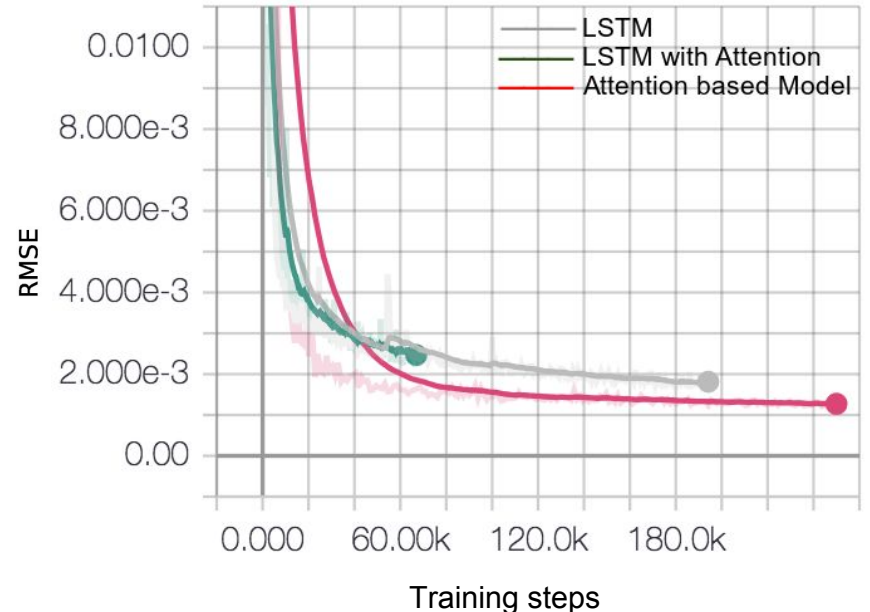

Figure 6: RMSE with predicting 60 steps (2 seconds)

degree videos from 5 categories. Data traces contain FoV samples varying from 1,000 to 4,000 in length. The video frame rate is about $30 \mathrm{fps}$. Video duration varies from one minutes to about two minutes. IMT and Tsinghua datasets are collected through HMD while users watch 360 degree videos. The IMT dataset includes data collected from 59 users watching five 70 seconds long 360-degree videos. The Tsinghua dataset includes data collected from 48 users watching 18 spherical videos from 5 categories and with video duration varying from 2 minutes 44 seconds to 10 minutes 55 seconds.

As for the experimental setup, we use Nvidia Tesla P100 GPUs and implement all models in Python using Tensorflow.

\section{RESULTS AND DISCUSSION}

The experiment results are shown as follows. Figure 6 shows the fast RMSE convergence performance of the attention based encoderdecoder network compared with LSTM and LSTM with attention models. It uses the IMT dataset i.e. 360-Degree Head Movement Dataset [7] for predicting 60 steps ahead given 300 time steps of past FoV data points. (The datapoint at each step is the FoV recording for each video frame at frame rate of 30 frames-per-second.) The solid curves are the smoothed average, while the shallow curves are instantaneous values.

Figure 7 shows the RMSE performance of attention-base model etc. at different numbers of prediction steps ahead. It compares Linear Regression, Truncated Linear Regression, LSTM, LSTM with attention, and attention-based encoder-decoder for the NYU dataset. The prediction is done for the future $20,40,60,80,100$ time steps which are corresponding to about $0.6,1.2,1.8,2.4,3.0$ seconds, each time step is about $30 \mathrm{~ms}$, given 300 time steps of past FoV data points. As we can see clearly that neural network models outperform Linear Regression models by a large margin, we omit the linear models in the following figures to better compare the performance of LSTM, LSTM with attention and Attention-base encoder-decoder. Figures 8, 9 and 10 show the RMSE performance of LSTM, LSTM with attention and attention-based encoder-decoder networks for NYU dataset, IMT dataset and Tsinghua dataset. The

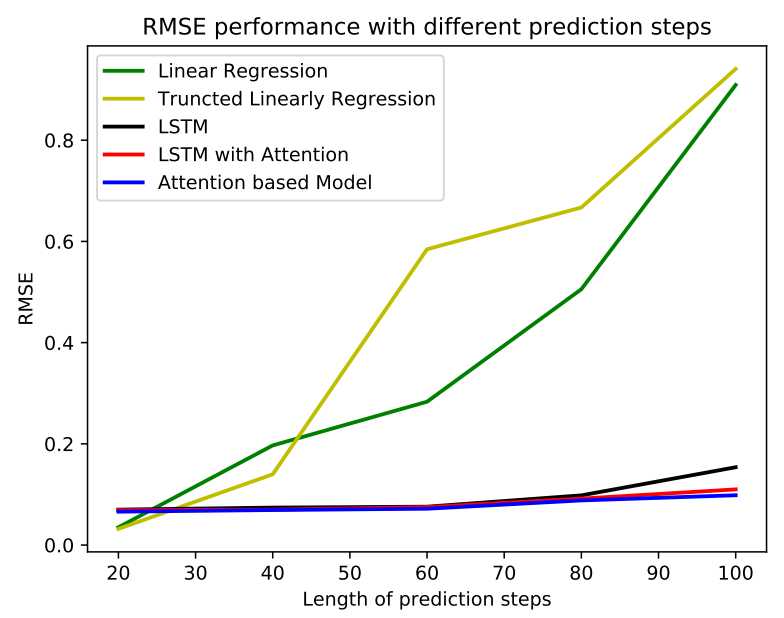

Figure 7: RMSE vs number of predictions: NYU dataset

attention-based encoder-decoder network outperforms the other two models significantly in all three datasets.

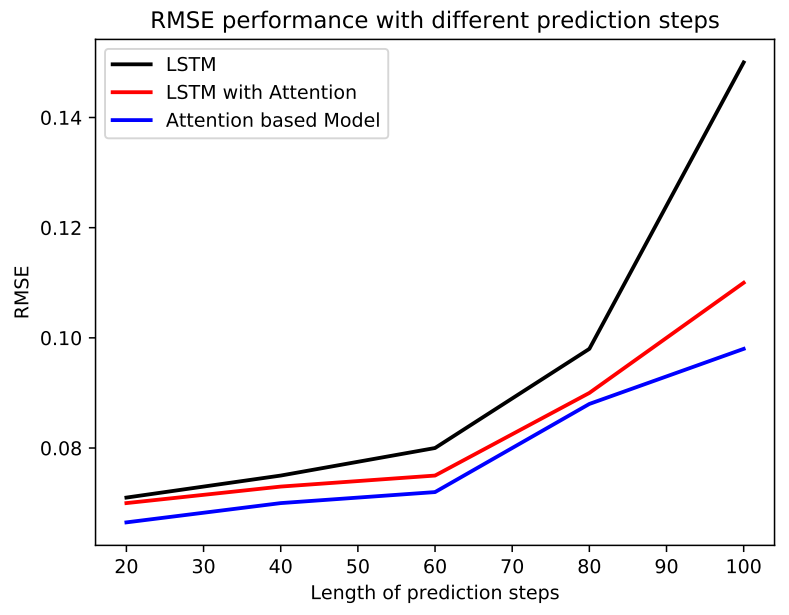

Figure 8: RMSE performance: NYU Dataset

We plot the training throughput performance of the Attentionbased model, LSTM, and LSTM with Attention in blue, green and red colors in Figure 11. The y axis is the number of training iterations per second. It shows that the attention-based model achieves the highest training throughput, almost twice as fast as the LSTM model and five times as the LSTM with attention, across the three datasets.

These results demonstrate that the attention based encoderdecoder networks achieves much better prediction accuracy, and faster training time compared with LSTM Models in the FoV prediction for 360-degree videos. 


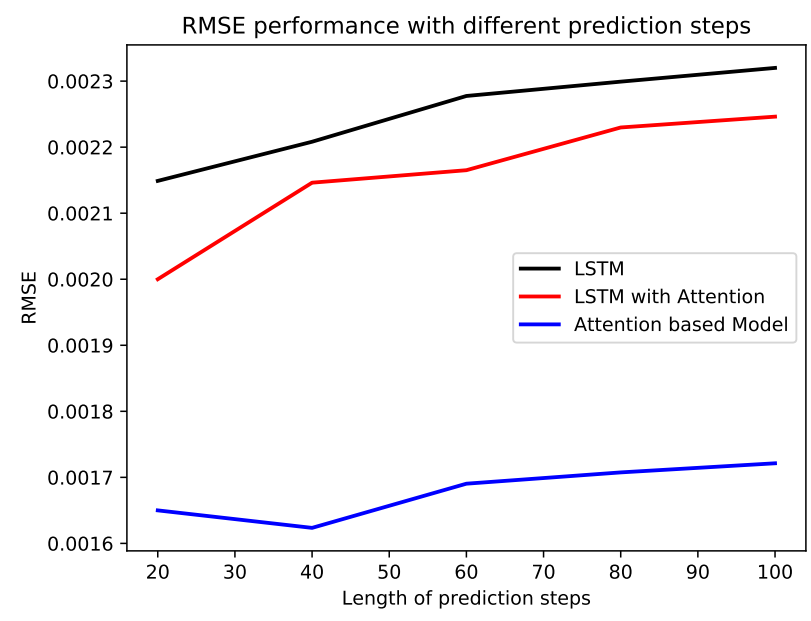

Figure 9: RMSE performance: IMT Dataset

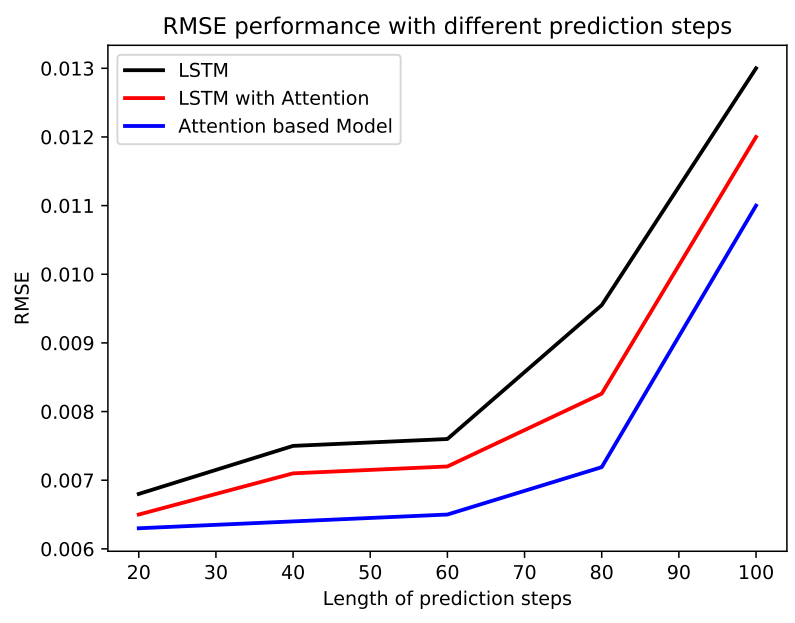

Figure 10: RMSE performance: Tsinghua Dataset

\section{CONCLUSION AND FUTURE WORK}

In this paper, we propose using novel attention-based neural encoderdecoder networks to predict FoVs in 360 degree videos. Our method outperforms linear regression models and LSTM models in accuracy and training throughtput on several 360-degree Head Movement datasets. Its low RMSE and high training throughput performance bring the benefits of reduced bandwidth usage and latency in delivering 360 degree videos for VR/AR applications. Our future work includes research on utilizing multi-modalities, such as video, audio and transcripts in 360-degree videos to further improve accuracy.

\section{REFERENCES}

[1] Alexandre Alahi, Kratarth Goel, Vignesh Ramanathan, Alexandre Robicquet, Li Fei-Fei, and Silvio Savarese. 2016. Social LSTM: Human Trajectory Prediction in Crowded Spaces. In The IEEE Conference on Computer Vision and Pattern Recognition (CVPR).

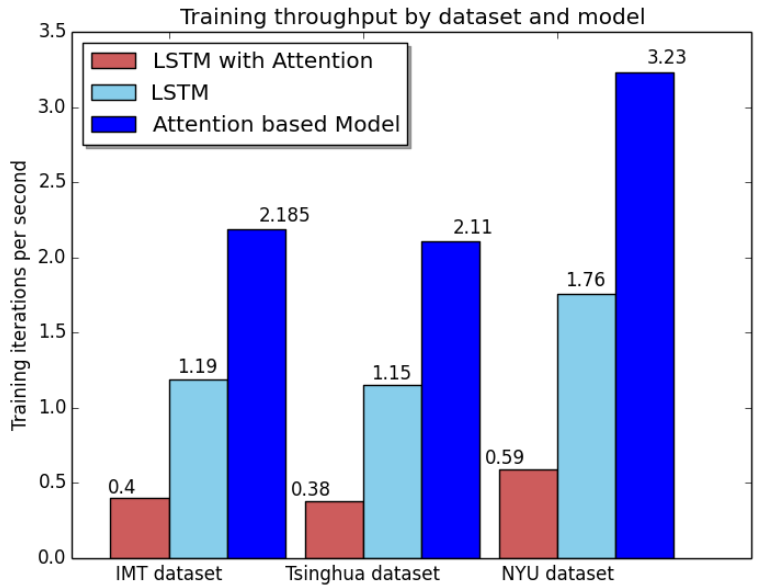

Figure 11: Training Throughput

[2] Lei Jimmy Ba, Ryan Kiros, and Geoffrey E. Hinton. 2016. Layer Normalization. CoRR abs/1607.06450 (2016). arXiv:1607.06450

[3] Dzmitry Bahdanau, Kyunghyun Cho, and Yoshua Bengio. 2014. Neural Machine Translation by Jointly Learning to Align and Translate. CoRR abs/1409.0473 (2014). arXiv:1409.0473

[4] Yanan Bao, Huasen Wu, Tianxiao Zhang, Albara Ah Ramli, and Xin Liu. 2016. Shooting a moving target: Motion-prediction-based transmission for 360-degree videos. In IEEE Big Data 2016. 1161-1170.

[5] Y. Bengio, P. Simard, and P. Frasconi. 1994. Learning long-term dependencies with gradient descent is difficult. IEEE Transactions on Neural Networks 5, 2 (March 1994), 157-166.

[6] Kyunghyun Cho, Bart van Merrienboer, ÃĞaglar GülÃ ̆gehre, Dzmitry Bahdanau, Fethi Bougares, Holger Schwenk, and Yoshua Bengio. 2014. Learning Phrase Representations using RNN Encoder-Decoder for Statistical Machine Translation. In $E M N L P$.

[7] Xavier Corbillon, Francesca De Simone, and Gwendal Simon. 2017. 360Degreee Video Head Movement Dataset. In Proceedings of the 8th ACM on Multimedia Systems Conference. New York, NY, USA, 199-204.

[8] S. Liu S. Srinivasan F. Duanmu, Y. Mao and Y. Wang. 2018. A Subjective Study of Viewer Navigation Behaviors When Watching 360-degree Videos on Computers. Proc. of IEEE International Conference on Multimedia Expo (ICME), San Diego, California, USA, 2018

[9] Kaiming He, Xiangyu Zhang, Shaoqing Ren, and Jian Sun. 2016. Deep Residual Learning for Image Recognition. In The IEEE Conference on Computer Vision and Pattern Recognition (CVPR).

[10] Sepp Hochreiter and Jürgen Schmidhuber. 1997. Long Short-Term Memory. Neural Comput. 9, 8 (Nov. 1997), 1735-1780.

[11] Huawei. 2016. Whitepaper on the VR-Oriented Bearer Network Requirement. http://www-file.huawei.com/-/media/CORPORATE/PDF/white20paper/ whitepaper-on-the-vr-oriented-bearer-network-requirement.pdf

[12] Ilya Sutskever, Oriol Vinyals, and Quoc V Le. 2014. Sequence to Sequence Learning with Neural Networks. In Advances in Neural Information Processing Systems. 3104-3112.

[13] Ashish Vaswani, Noam Shazeer, Niki Parmar, Jakob Uszkoreit, Llion Jones, Aidan N. Gomez, Lukasz Kaiser, and Illia Polosukhin. 2017. Attention is All You Need. https://arxiv.org/pdf/1706.03762.pdf

[14] Chenglei Wu, Zhihao Tan, Zhi Wang, and Shiqiang Yang. 2017. A Dataset for Exploring User Behaviors in VR Spherical Video Streaming. In Proceedings of the 8th ACM on Multimedia Systems Conference (MMSys'17). ACM, New York, NY, USA, 193-198.

[15] Yanyu Xu, Yanbing Dong, Junru Wu, Zhengzhong Sun, Zhiru Shi, Jingyi Yu, and Shenghua Gao. 2018. Gaze Prediction in Dynamic 360Âř Immersive Videos. In The IEEE Conference on Computer Vision and Pattern Recognition (CVPR).

[16] Jianzhong Zhang Madhukar Budagavi Xueshi Hou, Sujit Dey. 2018. Predictive View Generation to Enable Mobile 360-degree and VR Experiences. ACM SIGCOMM VR/AR workshop (2018).

[17] Zhimin Xu Xinggong Zhang Zongming Guo Yixuan Ban, Lan Xie. 2018. CUB360: Exploiting Cross-Users Behaviors for Viewport Prediction in 360 Video Adaptive Streaming. IEEE ICME 2018, San Diego, California, USA. 